\title{
Noncovariant Lagrangians Are Presented Which Yield Two-Component Equations of Motion for a Class of Relativistic Mechanical Systems in 1 + 1 Dimensions Including the Harmonic Oscillator
}

\author{
Robert L. Anderson \\ Department of Physics and Astronomy, University of Georgia, Athens, Georgia, USA \\ Email: andersonrobert1066@gmail.com
}

How to cite this paper: Anderson, R.L. (2020) Noncovariant Lagrangians Are Presented Which Yield Two-Component Equations of Motion for a Class of Relativistic Mechanical Systems in $1+1$ Dimensions Including the Harmonic Oscillator. Applied Mathematics, 11, 917-921. https://doi.org/10.4236/am.2020.119059

Received: August 1, 2020

Accepted: September 13, 2020

Published: September 16, 2020

Copyright ( 2020 by author(s) and Scientific Research Publishing Inc. This work is licensed under the Creative Commons Attribution International License (CC BY 4.0).

http://creativecommons.org/licenses/by/4.0/ (c) (i) Open Access

\begin{abstract}
First, a Lagrangian is presented and authenticated for a Relativistic Harmonic Oscillator in $1+1$ dimensions. It yields a two-component set of equations of motion. The time-component is the missing piece in all previous discussions of this system! The second result is that this Oscillator Langrangian generalizes to Langrangians for a class of particles in $1+1$ dimensions subject to an arbitrary potential $V$ which is space dependent only.
\end{abstract}

\section{Keywords}

Harmonic Oscillator

\section{Introduction}

All relativistic particles treated in this paper are in $1+1$ dimensions with the convention $\left(x^{\mu}\right)=\left(x^{o}, x^{1}\right)=\left(c t, x^{1}\right)$, where $\mathrm{d} \tau=\mathrm{d} t \sqrt{1-\left(\frac{1}{c} \frac{\mathrm{d} x^{1}}{\mathrm{~d} t}\right)^{2}}, \quad \tau \quad$ is the proper time.

We present for the first time a Langrangian for the Relativistic Harmonic Oscillator with potential energy $V\left(x^{1}\right)=\frac{1}{2} k\left(x^{1}\right)^{2}$.

The Langrangian is presented in Section 2 and authenticated in Section 3 for a Relativistic Harmonic Oscillator in $1+1$ dimensions. It yields a two-component set of equations of motion. The time-component is the missing piece in all pre- 
vious discussions of this system! Further, it sets the fundamental work of Goldstein [1] in the context of a complete theoretical treatment of this system. It also establishes the importance of MacColl [2] using the time-component of what is equivalent to the equations of motion in order to numerically solve for the trajectory of the Relativistic Oscillator.

In Section 4 we parallel the development for the Relativistic Harmonic Oscillator for a class of relativistic particles in $1+1$ dimensions each described by potential energy which is only space dependent.

\section{A Relativistic Oscillator Lagrangian}

We now present for the first time a Langrangian for the Relativistic Harmonic Oscillator with potential energy $V\left(x^{1}\right)=\frac{1}{2} k\left(x^{1}\right)^{2}$ :

$$
L^{(\tau)}=\frac{m_{0} c}{2} \frac{\mathrm{d} x^{\mu}}{\mathrm{d} \tau} g_{\mu \nu} \frac{\mathrm{d} x^{v}}{\mathrm{~d} \tau}+\frac{\mathrm{d} x^{o}}{\mathrm{~d} \tau} \frac{1}{2} k\left(x^{1}\right)^{2},
$$

where $\left[g_{\mu v}\right]=\left[\begin{array}{cc}1 & 0 \\ 0 & -1\end{array}\right]$ and $\frac{\mathrm{d} x^{\mu}}{\mathrm{d} \tau} g_{\mu \nu} \frac{\mathrm{d} x^{\nu}}{\mathrm{d} \tau}=\frac{\mathrm{d} x^{o}}{\mathrm{~d} \tau} \frac{\mathrm{d} x^{o}}{\mathrm{~d} \tau}-\frac{\mathrm{d} x^{1}}{\mathrm{~d} \tau} \frac{\mathrm{d} x^{1}}{\mathrm{~d} \tau}$.

It is noncovariant and holds only in the frame in which the oscillator is first set in motion consistent with Equation (4).

\section{Authentication of the Oscillator Lagrangian}

Lagrange's two-component equation of motion follows from the variation of (1)

$$
\frac{\partial L^{(\tau)}}{\partial x^{\mu}}-\frac{\mathrm{d}}{\mathrm{d} \tau} \frac{\partial L^{(\tau)}}{\partial\left(\frac{\mathrm{d} x^{\mu}}{\mathrm{d} \tau}\right)}=0, \quad \mu=0,1 .
$$

This implies the following two equations of motion

$$
\begin{gathered}
(\mu=0) \frac{\mathrm{d}}{\mathrm{d} \tau}\left(\frac{1}{2} k\left(x^{1}\right)^{2}\right)+m_{0} c \frac{\mathrm{d}}{\mathrm{d} \tau}\left(\frac{\mathrm{d} x^{0}}{\mathrm{~d} \tau}\right)=0, \\
(\mu=1) \frac{\mathrm{d} x^{0}}{\mathrm{~d} \tau}\left(k x^{1}\right)+m_{0} c\left(\frac{\mathrm{d}}{\mathrm{d} \tau} \frac{\mathrm{d} x^{1}}{\mathrm{~d} \tau}\right)=0 .
\end{gathered}
$$

Equation (3a) is the time-component of the equations of motion which was missing from all prior discussions of the Relativistic Harmonic Oscillator System! We now proceed to derive its content and explore its consequences.

Equation (3a) yields

$$
E-\frac{1}{2} k\left(x^{1}\right)^{2}-\frac{m_{0} c^{2}}{\sqrt{1-\left(\frac{1}{c} \frac{\mathrm{d} x^{1}}{\mathrm{~d} t}\right)^{2}}}=0,
$$

where $E$ is a constant, or rearranging 


$$
E=\frac{m_{0} c^{2}}{\sqrt{1-\left(\frac{1}{c} \frac{\mathrm{d} x^{1}}{\mathrm{~d} t}\right)^{2}}}+\frac{1}{2} k\left(x^{1}\right)^{2}
$$

The term $\frac{m_{0} c^{2}}{\sqrt{1-\left(\frac{1}{c} \frac{\mathrm{d} x^{1}}{\mathrm{~d} t}\right)^{2}}}$ is the rest energy $m_{0} c^{2}$ scaled by the motion and $\frac{1}{2} k\left(x^{1}\right)^{2}$ is the potential energy. Thus, $E$ is the total energy of the Harmonic Oscillator. Hence, the content of the $\mu=0$ equation of motion is the conservation of energy. See MacColl [2] Equation (1.2).

With (4) in hand, we first return to (3a)

$$
k x^{1} \frac{\mathrm{d} x^{1}}{\mathrm{~d} \tau}+\frac{\mathrm{d}}{\mathrm{d} \tau}\left(\frac{\mathrm{d}}{\mathrm{d} \tau} m_{0} c x^{0}\right)=0
$$

or

$$
\frac{\mathrm{d}}{\mathrm{d} \tau}\left(m_{0} c \frac{\mathrm{d} x^{0}}{\mathrm{~d} \tau}\right)=-k x^{1} \frac{\mathrm{d} x^{1}}{\mathrm{~d} \tau},
$$

or

$$
\frac{\mathrm{d}}{\mathrm{d} \tau}\left(\frac{m_{0} c^{2}}{\sqrt{1-\left(\frac{\mathrm{d} x^{1}}{c \mathrm{~d} t}\right)^{2}}}\right)=-k x^{1} \frac{\mathrm{d} x^{1}}{\mathrm{~d} \tau}
$$

or

$$
\frac{\mathrm{d}}{\mathrm{d} t}\left(\frac{m_{0} c^{2}}{\sqrt{1-\left(\frac{\mathrm{d} x^{1}}{c \mathrm{~d} t}\right)^{2}}}\right)=-k x^{1} \frac{\mathrm{d} x^{1}}{\mathrm{~d} t} .
$$

(Compare with Goldstein's [1] Equation (7.95). Note Goldstein uses the old notation involving $i=\sqrt{-1}$ and we are only using (7.82) where $V$ is the harmonic oscillator potential.)

We now turn to Equation (3b) which we can now rewrite as

$$
\frac{1}{\sqrt{1-\left(\frac{1}{c} \frac{\mathrm{d} x^{1}}{\mathrm{~d} t}\right)^{2}}} \frac{\mathrm{d}}{\mathrm{d} t}\left(\frac{m_{0} \frac{\mathrm{d} x^{1}}{\mathrm{~d} t}}{\sqrt{1-\left(\frac{1}{c} \frac{\mathrm{d} x^{1}}{\mathrm{~d} t}\right)^{2}}}\right)=\frac{-k x^{1}}{\sqrt{1-\left(\frac{1}{c} \frac{\mathrm{d} x^{1}}{\mathrm{~d} t}\right)^{2}}}
$$

or equivalently 


$$
\frac{\mathrm{d}}{\mathrm{d} t}\left(\frac{m_{0} \frac{\mathrm{d} x^{1}}{\mathrm{~d} t}}{\sqrt{1-\left(\frac{1}{c} \frac{\mathrm{d} x^{1}}{\mathrm{~d} t}\right)^{2}}}\right)=-k x^{1}
$$

Equation (6a) is Equation (7.83) with Equation (7.87) in Goldstein [1]. (Remember, Goldstein did all this without a Lagrangian which could yield the two component Lagrangian equations of motion.)

Equation (6b) follows the original Einsteinian prescription for a point particle in a potential $V\left(x^{1}\right)$. One takes the non-relativistic equation of motion and modifies it only by replacing the mass by $m_{0} / \sqrt{1-\left(\frac{\mathrm{d} x^{1}}{c \mathrm{~d} t}\right)^{2}}$ where $m_{0}$ is the rest mass.

This was the equation of motion that MacColl assumed. He then multiplied it by $\frac{\mathrm{d} x^{1}}{\mathrm{~d} t}$ and integrated to obtain the energy $E$ (he used scaling). He solved this for $\frac{\mathrm{d} x^{1}}{\mathrm{~d} t}$. Solving for $\mathrm{d} t$ via a change of variables and setting initial conditions, he arrived at an expression for $t$ in terms of incomplete elliptic integrals. This he solved numerically. Our summary of MacColl's approach in our notation is contained in [3].

\section{Lagrangians for a Class of Relativistic Particles}

Finally, we parallel the development for the Relativistic Harmonic Oscillator in an abbreviated form because the logic is the same for a class of relativistic particles in $1+1$ dimensions described by a potential energy $V\left(y^{1}\right)$, where we replace $\left(x^{\mu}\right)=\left(x^{o}, x^{1}\right)$ with $\left(y^{\mu}\right)=\left(y^{o}, y^{1}\right)$.

Then we have

$$
L^{(\tau)}=\frac{m_{0} c}{2} \frac{\mathrm{d} y^{\mu}}{\mathrm{d} \tau} g_{\mu \nu} \frac{\mathrm{d} y^{v}}{\mathrm{~d} \tau}+\frac{\mathrm{d} y^{o}}{\mathrm{~d} \tau} V\left(y^{1}\right) .
$$

This is noncovariant and holds in the frame in which $V\left(y^{1}\right)$ is turned on consistent with Equation (9).

This implies

$$
\begin{gathered}
(\mu=0) \frac{\mathrm{d}}{\mathrm{d} \tau}\left(V\left(y^{1}\right)+m_{0} c \frac{\mathrm{d} y^{0}}{\mathrm{~d} \tau}\right)=0, \\
(\mu=1) \frac{\mathrm{d} y^{o}}{\mathrm{~d} \tau}\left(\frac{\partial V\left(y^{1}\right)}{\partial y^{1}}\right)+m_{0} c\left(\frac{\mathrm{d}}{\mathrm{d} \tau} \frac{\mathrm{d} y^{1}}{\mathrm{~d} \tau}\right)=0 .
\end{gathered}
$$

(8a) implies conservation of energy $E$

$$
E=\frac{m_{0} c^{2}}{\sqrt{1-\left(\frac{1}{c} \frac{\mathrm{d} y^{1}}{\mathrm{~d} t}\right)^{2}}}+V\left(y^{1}\right) .
$$


Equation (8a) can be rewritten as

$$
\frac{\mathrm{d}}{\mathrm{d} t}\left(\frac{m_{0} c^{2}}{\sqrt{1-\left(\frac{1}{c} \frac{\mathrm{d} y^{1}}{\mathrm{~d} t}\right)^{2}}}\right)=-\frac{\mathrm{d}}{\mathrm{d} t} V\left(y^{1}\right) .
$$

Equation (8b) can be rewritten as

$$
\frac{\mathrm{d}}{\mathrm{d} t}\left(\frac{m_{0} \frac{\mathrm{d} y^{1}}{\mathrm{~d} t}}{\sqrt{1-\left(\frac{1}{c} \frac{\mathrm{d} y^{1}}{\mathrm{~d} t}\right)^{2}}}\right)=-\frac{\partial V\left(y^{1}\right)}{\partial y^{1}} .
$$

which again fits the Einsteinian prescription. Thus the theoretical foundations are complete for all relativistic point particles governed by a potential energy $V\left(y^{1}\right)$ in $1+1$ dimensions.

Examples are provided by the hierarchy described in [3] and [4] for which $V\left(x^{1}\right)=-\left.\frac{k_{2 n}}{2 n}\left(x_{2 n}^{1}\right)^{2 n}\right|_{n>1}$. The notation $\left(y^{o}, y^{1}\right)$ is replaced by $\left(x_{2 n}^{o}, x_{2 n}^{1}\right)$ for the $n$th member of the hierarchy. $n=1$ is the Harmonic Oscillator discussed in the first part of this paper.

\section{Acknowledgements}

I would like to dedicate this paper to Nail Ibragimov-Eminent Professor, Mathematician and friend. Also I would like to acknowledge Professor Herbert Goldstein's contribution to this paper. His work in Chapter 7 of his book [1] inspired this paper. Finally to my wife, Dianne, thanks for her enduring support.

\section{Conflicts of Interest}

The author declares no conflicts of interest regarding the publication of this paper.

\section{References}

[1] Goldstein, H. (1980) Classical Mechanics. 2nd Edition, Addison-Wesley Series in Physics, Boston.

[2] MacColl, L.A. (1957) Theory of the Relativistic Oscillator. American Journal of Physics, 25, 535. https://doi.org/10.1119/1.1934543

[3] Anderson, R.L. (2014) Linearization of the Relativisitic Oscillator Hierarchy. http://arxiv.org/1605.02107

[4] Anderson, R.L. (2010) An Invertible Linearization Map for the Quartic Oscillator. $J M P$, 51, 122904. https://doi.org/10.1063/1.3527070 